\title{
Intrapartum Stillbirth
}

National Cancer Institute

\section{Source}

National Cancer Institute. Intrapartum Stillbirth. NCI Thesaurus. Code C113382.

Fetal death greater than or equal to 20 weeks of gestation during labor with Apgar scores of 0 at 1 minute, 5 minutes and beyond. 